\title{
Identification and characterization of orthologs of AtNHX5 and AtNHX6 in Brassica napus
}

\section{Brett A. Ford, Joanne R. Ernest and Anthony R. Gendall*}

Department of Botany, La Trobe University, Melbourne, VIC, Australia

\section{Edited by:}

Xiaowu Wang, Chinese Academy of Agricultural Sciences, China

Reviewed by:

Kees Venema, Consejo Superior de Investigaciones Cientificas, Spain

Xiaowu Wang, Chinese Academy of Agricultural Sciences, China

\section{${ }^{*}$ Correspondence:}

Anthony R. Gendall, Department of

Botany, La Trobe University,

Bundoora, Melbourne, VIC,

Australia.

e-mail: t.gendall@latrobe.edu.au
Improving crop species by breeding for salt tolerance or introducing salt tolerant traits is one method of increasing crop yields in saline affected areas. Extensive studies of the model plant species Arabidopsis thaliana has led to the availability of substantial information regarding the function and importance of many genes involved in salt tolerance. However, the identification and characterization of $A$. thaliana orthologs in species such as Brassica napus (oilseed rape) can prove difficult due to the significant genomic changes that have occurred since their divergence approximately 20 million years ago (MYA). The recently released Brassica rapa genome provides an excellent resource for comparative studies of $A$. thaliana and the cultivated Brassica species, and facilitates the identification of Brassica species orthologs which may be of agronomic importance. Sodium hydrogen antiporter (NHX) proteins transport a sodium or potassium ion in exchange for a hydrogen ion in the other direction across a membrane. In $A$. thaliana there are eight members of the NHX family, designated AtNHX1-8, that can be sub-divided into three clades, based on their subcellular localization: plasma membrane (PM), intracellular class I (IC-I) and intracellular class II (IC-II). In plants, many NHX proteins are primary determinants of salt tolerance and act by transporting $\mathrm{Na}^{+}$out of the cytosol where it would otherwise accumulate to toxic levels. Significant work has been done to determine the role of both PM and IC-I clade members in salt tolerance in a variety of plant species, but relatively little analysis has been described for the IC-II clade. Here we describe the identification of B. napus orthologs of AtNHX5 and AtNHX6, using the B. rapa genome sequence, macro- and micro-synteny analysis, comparative expression and promoter motif analysis, and highlight the value of these multiple approaches for identifying true orthologs in closely related species with multiple paralogs.

Keywords: Arabidopsis, NHX, antiporter, Brassica, sodium transport, potassium transport, pH, cation transport

\section{INTRODUCTION}

It is estimated that more than 800 million hectares of land worldwide and approximately $20 \%$ of irrigated farmland are negatively impacted by salinity (FAO, 2009). While there are many different salts that contribute to the salinization of a landscape, by far the most abundant and damaging is $\mathrm{NaCl}$ (Tester and Davenport, 2003). $\mathrm{NaCl}$ in the soil inhibits plant growth by causing an initial osmotic stress, followed by the accumulation of $\mathrm{Na}^{+}$ions in the plant to toxic levels (Munns and Tester, 2008). Improved crop yield under saline conditions can be achieved by identifying genes which confer salt tolerance, and introducing them to crop species by traditional breeding or transgenesis.

In plants, members of the monovalent cation/proton antiporter (CPA1) gene family exchange a sodium, potassium or lithium ion for a hydrogen ion across a cellular membrane. They can be classified into two distinct sub families, the plasma membrane (PM) localized NHAP family and the intracellular localized (IC) NHX family (Brett et al., 2005a; Chanroj et al., 2012). These proteins are important in maintaining $\mathrm{pH}$ and ion homeostasis, and are conserved across all phyla and kingdoms (Brett et al., 2005a). In Arabidopsis thaliana, there are two members of the
NHAP family (SOS1/AtNHX7 and AtNHX8) and six members of the IC-NHX family, designated AtNHX1-6 (Brett et al., 2005a; Chanroj et al., 2012). The IC-NHX family can be further subdivided into two clades based on their cellular localization: the IC-I clade (AtNHX1-4), localized to the tonoplast; or the IC-II clade (AtNHX5-6), localized to endosomal compartments (Brett et al., 2005a; Bassil et al., 2011). In plants, NHX proteins are important determinants of $\mathrm{Na}^{+}$tolerance, detoxifying cytosolic $\mathrm{Na}^{+}$by transportation out of the cell to the apoplastic space, or by sequestration into subcellular compartments (Apse et al., 1999; Shi et al., 2003; Rodriguez-Rosales et al., 2008). Members of the PM (AtNHX7/SOS1) and IC-I (AtNHX1) clades have been shown to be upregulated in response to $\mathrm{NaCl}$, and to confer salt tolerance upon over-expression in A. thaliana (Apse et al., 1999; Shi et al., 2002, 2003; Shi and Zhu, 2002). Of the IC-II clade, AtNHX5 and the rice ortholog OsNHX5 are both up regulated in response to $\mathrm{NaCl}$ (Yokoi et al., 2002; Fukuda et al., 2011) and over expressing AtNHX5 in a variety of plant species including rice (Li et al., 2011) and tomato (Rodriguez-Rosales et al., 2008) has been shown to increase their tolerance to $\mathrm{NaCl}$. Recent work has shown that AtNHX5 and AtNHX6 are functionally redundant, 
are important for normal plant growth and development, are endosome associated and have an important role in protein trafficking to the vacuole (Bassil et al., 2011). A similar role in protein trafficking has been demonstrated for the yeast ortholog ScNHX1 (Bowers et al., 2000; Brett et al., 2005b). Interestingly, comparatively little work has been undertaken investigating the role of the Class II NHX proteins from important crop species in $\mathrm{NaCl}$ tolerance.

Brassica napus is a member of the Brassicaceae family and is an important oil crop species. B. napus cultivation has increased significantly over recent years and it is now the second largest crop for the production of oil seeds and oil meals, and the third largest crop used in the production of vegetable oil (USDA, 2009). As both $A$. thaliana and $B$. napus are members of the family Brassicaceae and therefore relatively closely related, a comparative study could be undertaken to try to identify the homologs of AtNHX5 and AtNHX6 to assess their importance in salt tolerance. However, there have been extensive large- and small-scale genome changes in the A. thaliana and B. napus genomes since their divergence approximately 13-17 million years ago (MYA) (Figure 1). $B$. napus is an allopolyploid species (AACC, $2 n=38$ ) arising from the hybridization of $B$. rapa (AA, $2 n=20)$ and Brassica oleracea (CC, $2 n=18$ ) (Figure 1). Additionally, all Brassica species appear to have undergone a whole genome triplication (WGT, $\mathrm{Br}-\alpha$ ) event since they diverged from $A$. thaliana (Figure 1). The A. thaliana genome has undergone extensive duplications, deletions, re-arrangements and a reduction in chromosome number even since its divergence from its close relative Arabidopsis lyrata only 5 MYA (Figure 1) (Hu et al., 2011). These confounding factors make direct comparative studies and homolog identification in $B$. napus based solely on A. thaliana genomic information problematic.

To overcome this problem, Schranz et al. (2006), building on the work of Parkin et al. (2005), have proposed that the comparative studies of $A$. thaliana and the Brassica species should be based upon an ancient karyotype of $n=8$, that is observed in at least $37 \%$ of the Brassicaceae species. The genome of this ancient karyotype can be further segmented into 24 conserved syntenic blocks (designated $\mathrm{A}-\mathrm{X}$ ) that can be identified in both the $A$. thaliana genome and the $B$. rapa (A genome) component of B. napus (Parkin et al., 2005; Schranz et al., 2006).

While this approach greatly aided comparative studies between A. thaliana and B. napus, the lack of high quality Brassica sequence still inhibited homolog identification. The recent release of the fully sequenced B. rapa genome (Wang et al., 2011) has enabled in-depth comparative studies between $A$. thaliana and B. napus.

Analysis of the $B$. rapa genome reveals that there has been significant gene loss since the most recent WGT (Wang et al., 2011). The triplicated $B$. rapa genome can be divided into three sub genomes based on their level of gene loss relative to A. thaliana, which have been designated the least fractionated genome (LF) with $30 \%$ gene loss, the medium fractionated genome (MF1) with 54\% gene loss and the most fractionated genome (MF2) with 64\% gene loss (Wang et al., 2011). However, analysis of the B. rapa genome shows that genes

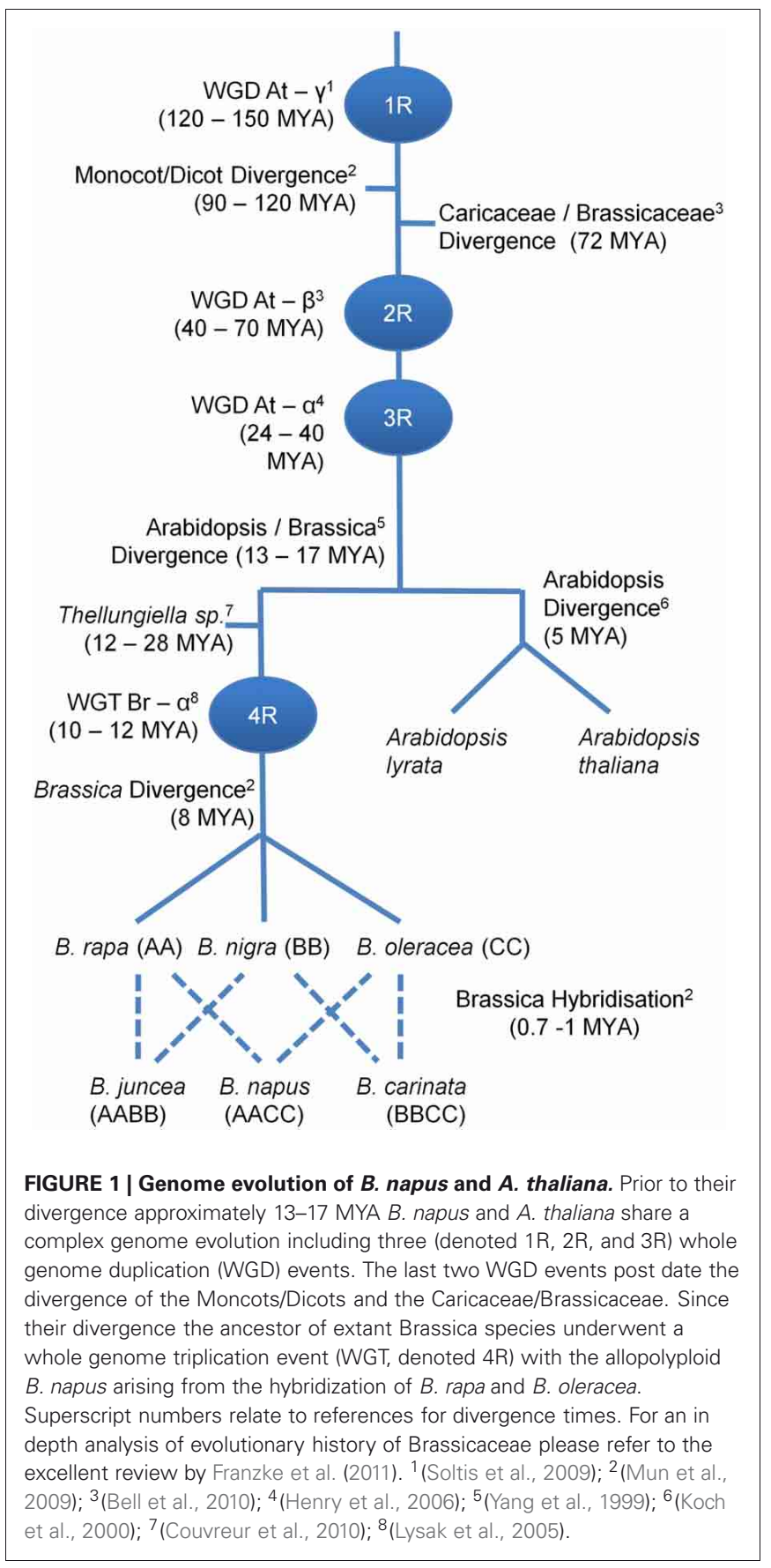

associated with response to environmental factors such as salt were over-retained (Wang et al., 2011) and as a result it is possible that there are three homologs of AtNHX5 and three homologs of AtNHX6 in B. rapa. As B. napus has both a $B$. rapa triplicated genome, and a triplicated $B$. oleracea $\mathrm{C}$ genome component there may be up to six AtNHX5 and six AtNHX6 homologs.

Here we describe the use of the $B$. rapa genomic sequence to aid in the clear identification of the B. napus homologs of AtNHX5 and AtNHX6 as a first step in evaluating their potential to improve salt tolerance. 


\section{MATERIALS AND METHODS IDENTIFICATION AND REANNOTATION OF B. rapa NHX GENES}

The initial identification of the complete set of $N H X$ genes in $B$. rapa used representative $A$. thaliana NHX protein sequences (See Table 1 for accession numbers) as query sequences in TBLASTN searches of the B. rapa genomic database in Phytozome version 8.0 (http://www.phytozome.org; Goodstein et al., 2012). Initial searches were performed using AtNHX1, AtNHX6, and AtSOS1/AtNHX7 amino acid sequences. Hits with an E-value of less than $10^{-45}$ were classified as putative NHXs and examined further. Preliminary alignments of $A$. thaliana NHX proteins to the annotated $B$. rapa proteins revealed some minor sequence dissimilarities in annotations that often corresponded to the lack of one or more exons in the $B$. rapa gene models, or from fusion of two adjacent genes into a single mis-annotated $\mathrm{BrNHX}$ gene. To refine these predictions, two approaches were used. Expressed sequence tag (EST) or cDNA sequences were identified by using the NHX amino acid sequences as the query for a TBLASTN search of all EST databases restricting the search to the taxid Brassica. The EST or cDNA sequences were then assigned to the relevant NHX gene based on the regions of maximum match and sequence alignment to the corresponding B. rapa genome. These sequences were aligned with the corresponding genomic region using Spidey (http://www.ncbi.nlm.nih.gov/IEB/ Research/Ostell/Spidey/index.html), using the "Plant" genomic sequence option and other default options. The identified exons were then incorporated into a final re-annotated open reading frame model. If no EST or cDNA support existed, amino acid sequences from the A. thaliana or other B. rapa ortholog corresponding to the mis-annotated regions were used as queries in a low stringency TBLASTN approach (expected E-value of 1.0), which allowed the correction of the original annotations. Gene models for BrNHX6.1 and BrNHX6.2 were generated using the FancyGene 1.4 gene model drawing program (http://www.bio. ieo.eu/fancygene Rambaldi and Ciccarelli, 2009). B. rapa NHX gene locations were determine by using the Brassica Database (http://brassicadb.org/brad).

\section{IDENTIFICATION OF Thellungiella parvula NHX GENES}

The identification of the intra-cellular NHX genes in T. parvula used representative Arabidopsis NHX protein sequences (See Table 1 for accession numbers) as query sequences in BLASTP searches of the T. parvula predicted gene models - protein version 2.0 database in the Thellungiella website [http://www.phytozome. org; (Dassanayake et al., 2011; Goodstein et al., 2012)]. Initial searches were performed using AtNHX1 and AtNHX6 amino acid sequences. Hits with an E value of less than $10^{-45}$ were classified as putative NHXs and examined further by phylogentic analysis.

\section{PHYLOGENETIC ANALYSIS}

The predicted amino acid sequences of all predicted B. rapa, T. parvula and $A$. thaliana NHXs were imported into MEGA5 (Tamura et al., 2011), and aligned using ClustalW with default parameters, and the corresponding amino acid alignments used for subsequent analyses. A neighbor-joining analysis was performed, using the Poisson model of amino acid substitution, with uniform rate among sites and pairwise deletion, and the phylogeny tested with 1000 bootstrap replications.

NHX6 nucleotide sequences from B. rapa, B. oleracea and B. napus and Brassica NHX6 ESTs from Genbank were imported into MEGA5 (Tamura et al., 2011) and aligned using Clustal W with default parameters. A neighbor-joining analysis was performed using the maximum composite likelihood method with uniform rate among sites and complete deletion, and the phylogeny tested with 1000 bootstrap replications.

\section{MICROSYNTENY OF AtNHX5 AND AtNHX6 AND THEIR CO-LINEARITY IN B. rapa}

AtNHX5 and AtNHX6 sequences were used as the query to search for homeologous regions in the three sub genomes in B. rapa using the syntenic paralogs search tool in the Brassica Database (http://brassicadb.org/brad). The region of A. thaliana chromosome 1 containing AtNHX6 and eight flanking genes upstream and downstream was then aligned to the three co-linear genomic segments from B. rapa. The region of $A$. thaliana chromosome 1 surrounding AtNHX5 was aligned in a similar manner, however due to the more complex nature of the co-linearity between this region and the corresponding $B$. rapa genomic segments the region of analysis was expanded to include 14 genes downstream and 12 genes upstream of AtNHX5.

\section{BrNHX6 PROMOTER ANALYSIS}

Nucleotide sequences of AtNHX6, BrNHX6.1, and BrNHX6.2, including $1119 \mathrm{bp}$ upstream of the predicted ATG start codon, and $500 \mathrm{bp}$ downstream of the predicted stop codon were obtained from Phytozome. Sequences were aligned and subjected to sliding window analysis of homology using VISualization Tool for Alignments (VISTA) web-based software (Frazer et al., 2004). Regions identified as highly conserved were analysed for conserved cis-regulatory elements (CRE) motifs using the Plant cisacting regulatory DNA elements (PLACE) database (Higo et al., 1999).

\section{PLANT GROWTH CONDITIONS}

Seeds of B. rapa (cv Chinese cabbage), B. oleracea (cv White cabbage) and $B$. napus (cv Westar) were sown direct to soil in seed raising mix and vermiculite (3:1) and watered twice a week. A general purpose fertilizer (Osmocote) was applied in liquid form every two weeks and plants were grown in a $16 \mathrm{~h}$ light: $8 \mathrm{~h}$ dark photoperiod at $22^{\circ} \mathrm{C}$.

For material used for qRT-PCR and isolation of cDNA, B. napus (cv Westar) seeds were surface sterilized by soaking in $70 \%$ ethanol for $5 \mathrm{~min}$ and $1 \%$ commercial bleach for $10 \mathrm{~min}$. Seeds were washed three times in sterile double distilled water and plated onto half strength MS plates (Murashige and Skoog, 1962) with $0 \mathrm{mM}$ or $200 \mathrm{mM} \mathrm{NaCl}$. Three replicate plates were made for each treatment. Seeds were stratified for $72 \mathrm{~h}$ and grown in a $16 \mathrm{~h}$ light: $8 \mathrm{~h}$ dark photoperiod at $22^{\circ} \mathrm{C}$.

\section{CLONING OF BRASSICA NHX6 ORF SEQUENCES}

Genomic DNA was extracted from B. rapa (cv Chinese cabbage) as described (Herrmann and Frischauf, 1987). RNA was extracted from eight week old leaf material of B. napus (cv Westar) and B. oleracea (cv white cabbage) and from two week 


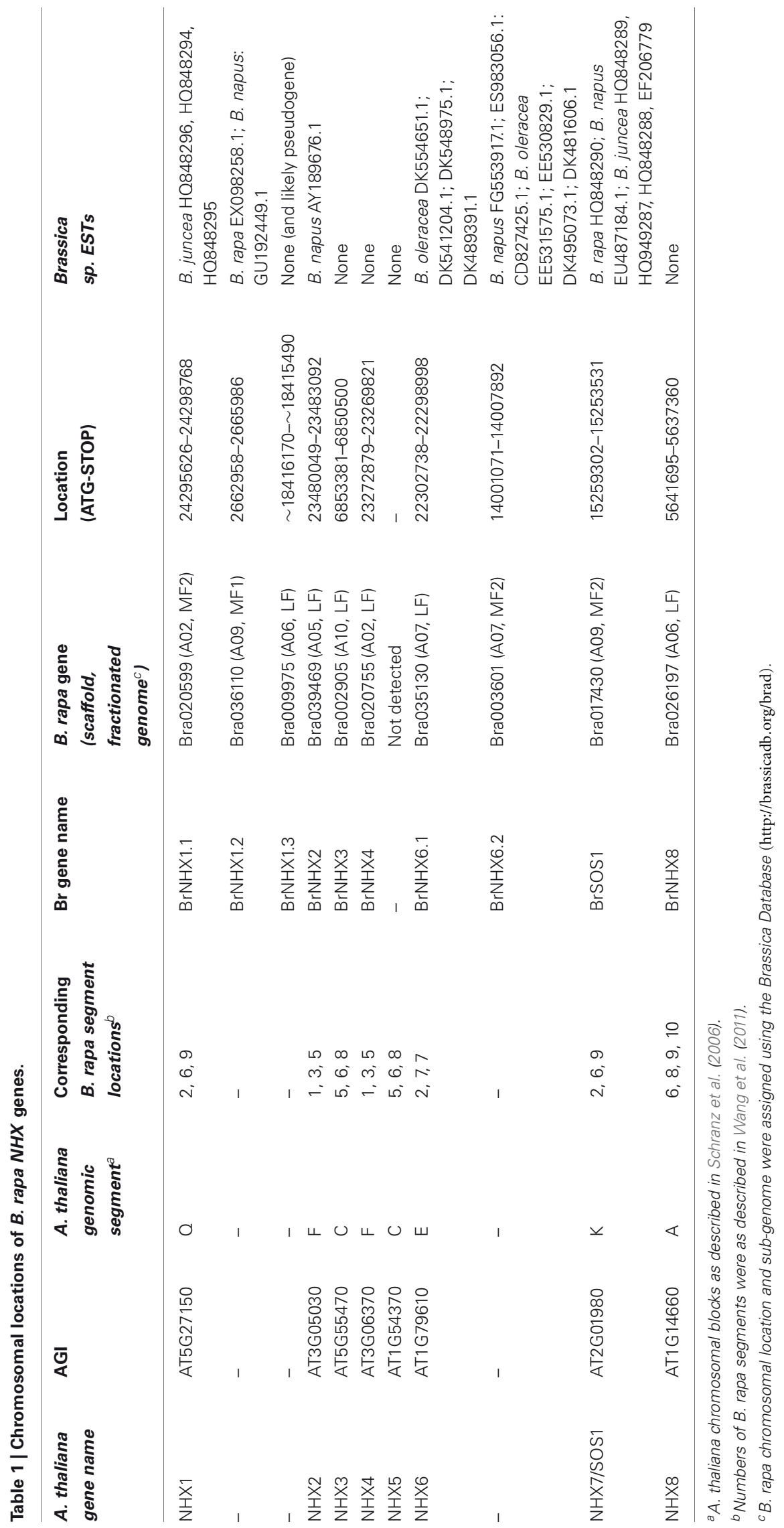


old whole seedlings B. napus (cv Westar) using the Qiagen RNeasy Kit (Qiagen \#74104) as per manufacturer's instructions. Approximately $500 \mathrm{ng}$ of RNA was used to synthesize cDNA using Superscript III (Life Technologies \#18080) following the manufacturer's recommendations, and the cDNA was diluted to a final volume of $100 \mu \mathrm{L}$ with sterile double distilled water.

Full length Brassica NHX6 ORF sequences were amplified by PCR from cDNA derived from eight week old leaf material of B. oleracea (Forward 5'-CACCATGTCGGAGATTTCGCCG-3' and Reverse 5' ${ }^{\prime}$-TAACCGGGGGCTAAATTTCTGA-3') and B. napus (Foward 5'-CACCATGTCGGAGATTTCGCCG-3' and Reverse $5^{\prime}$-TAACCGGGGGCTAAATTTCTGA- $\left.3^{\prime}\right)$. The PCR product was then cloned into the pENTR D/TOPO vector (Life Technologies \#K2435-20) as per manufacturer's instructions. Approximately 10 independent clones from each ligation were sequenced with one unique coding sequence being identified for both B. napus (Genbank JX082291) and B. oleracea (Genbank JX082292).

\section{IDENTIFICATION AND CLONING OF PARTIAL Brassica NHX6 SEQUENCES \\ Primer design}

The B. rapa NHX6.1 and NHX6.2 genes, the full length B. napus (JX082291) and B. oleracea (JX082292) ORF sequences as well as other $B$. napus. $B$. rapa and $B$. oleracea EST sequences that were available were aligned in Vector NTI v11.5 (Invitrogen). From this alignment regions of $100 \%$ nucleotide conservation between all divergent Brassica species sequences were identified and primers (Forward 5'-GCTTGAAGCCCTAGAGGTTGT-3' and Reverse 5'-CGTTATTACTTGTGAAGAACGTGTT-3') designed within these regions to amplify all potential $\mathrm{A}$ and $\mathrm{C}$ genome B. napus NHX6 homologs.

Using these primers, partial Brassica NHX6 sequences were amplified by PCR from cDNA of 2-week-old whole seedlings in B. napus and from genomic DNA in B. rapa. DNA from the PCR was purified using the Promega Wizard PCR clean up kit (Promega \#A9282) and then cloned into pGEM T-easy vector (Promega \#A1360) as per manufacturer's instructions. Approximately 10 independent clones from each ligation were sequenced using the M13_F sequencing primer.

\section{GENE EXPRESSION ANALYSIS}

Total RNA was extracted from whole seedlings of $B$. napus (cv Westar) two weeks after germination using the Qiagen RNeasy Kit (Qiagen \#74,104) as per manufacturer's instructions. Total RNA (2 $\mu \mathrm{g})$ was DNase treated with the Promega RQ1 DNase kit (Promega \#M6101) as per manufacturer's instructions to remove any genomic DNA contamination.

Primers were designed for the BnNHX6.1 gene (Forward 5'-CATCCTTTTCTCATTCTGTTCATCG-3' and Reverse $5^{\prime}$-TCGAAGTCCACTGTACCAAAG-3') and for the BnNHX6.2 gene (Forward 5'-GATAGCCGTGATACATCCCTTG- $3^{\prime}$ and Reverse $5^{\prime}$-AGTTCTGAAAATGACTTTGCGC-3') based on the corresponding BrNHX6.1 or BrNHX6.2 open reading frame sequences identified from the B. rapa genome.

Quantitative real-time PCR was performed using the BioRad iCycler and the iScript One Step RT-PCR kit with SYBR green (Bio-Rad \#170-8892) as per manufacturer's instructions. PCR conditions consisted of a reverse transcription step at $50^{\circ} \mathrm{C}$ for $10 \mathrm{~min}$, a reverse transcription inactivation step at $95^{\circ} \mathrm{C}$ for $5 \mathrm{~min}$ and 40 cycles of $95^{\circ} \mathrm{C}$ for $10 \mathrm{~s}$ followed by either $61^{\circ} \mathrm{C}$ $(B n N H X 6.1)$ or $60^{\circ} \mathrm{C}(B n N H X 6.2)$ for $30 \mathrm{~s}$. Results were visualized using BioRad iQ5 Optical System Software. A dissociation analysis was performed and the PCR products were sequenced to confirm PCR specificity to the $B n N H X 6$ transcript. Expression of the BnNHX6 genes was normalized relative to the expression of BnUBC_21 (Forward 5'-CCTCTGCAGCCTCCTCAAGT-3' and Reverse $5^{\prime}$-CATATCTCCCCTGTCTTGAAATGC-3') previously validated as a reference gene for qRT-PCR in $B$. napus (Chen et al., 2010). Transcript abundance was calculated using the Pfaffl model for relative quantification with efficiency correction (Pfaffl, 2004). All experiments were completed in triplicate on three independent biological replicates.

\section{RESULTS \\ IDENTIFICATION AND PHYLOGENETIC ANALYSIS OF B. rapa NHX GENES}

As a first step to identify the B. napus AtNHX5 and AtNHX6 homologs, we searched the now fully sequenced $B$. rapa genome. As AtNHX5 and AtNHX6 are very closely related to the other A. thaliana NHX members AtNHX1-4 and AtNHX78 , unmabiguous identification of the corresponding $B$. rapa homologs may be difficult. To clearly identify and unambiguously assign the AtNHX5 and AtNHX6 B. rapa homologs, we identified all the $N H X$ genes in the genome of $B$. rapa. To determine the complete complement of $N H X$ genes, we searched the B. rapa genome sequence and identified ten $N H X$ genes (hereafter called $B r N H X$; see Table 1 for complete details). A phylogenetic analysis of the corresponding predicted amino acid sequences of the encoded proteins revealed that $B$. rapa is likely to express five intracellular class one (IC-I) genes, two intracellular class two (IC-II) genes and two PM clade members (Figure 2A). Additional evidence of one pseudogene (BrNHX1.3) could also be detected. In some cases, multiple $B$. rapa genes could be detected that corresponded to a single $N H X$ gene in A. thaliana, but surprisingly, in only one case could three $B$. rapa orthologs corresponding to a AtNHX gene be detected (AtNHX1 and Br.NHX1.1, Br NHX1.2 and $B r N H X 1.3$; Table 1) suggesting substantial gene loss of $N H X$ genes in B. rapa following the most recent WGT. To further investigate this gene loss, we examined the chromosomal location of the $B$. rapa NHX genes, and compared these to the described fractionated genomes (Wang et al., 2011). Six of the $10 \mathrm{~B}$. rapa NHXs were retained on the LF sub-genome, one on the MF1 and three on the MF2 (Table 1). The proteins encoded by the two class II-IC NHX genes identified in B. rapa are clearly differentiated from other members of the A. thaliana NHX family (Figure 2A) and are likely to be the true orthologs of AtNHX5 and AtNHX6. Interestingly, both of the identified class II-IC NHX genes are more closely related to AtNHX6 than to AtNHX5 (Figure 2A) indicating that there may not be a $B$. rapa ortholog of AtNHX5.

To further investigate the apparent absence of a AtNHX5 ortholog in B. rapa, we decided to try to identify the intracellular NHX genes in the closely related species T. parvula. 

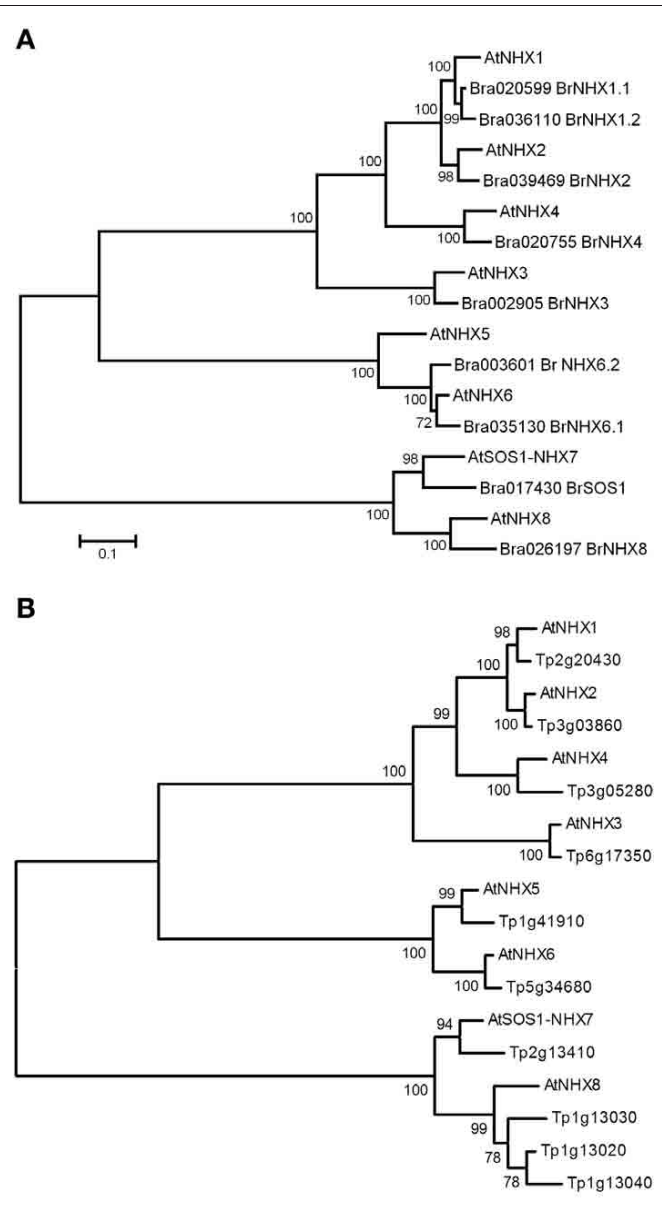

$\longmapsto .1$

FIGURE 2 | Phylogenetic analysis of $B$. rapa and $T$. parvula NHX proteins. Amino acid sequences of $\mathrm{NHX}$ proteins from B. rapa (A), T. parvula (B) and $A$. thaliana were aligned using Clustal $W$, and a Neighbor-joining tree generated. Numbers at nodes correspond to bootstrap support percentages following 1000 replications, and the scale is in amino acid changes per site.

T. parvula diverged from the Brassica species after the divergence from $A$. thaliana but before the $\mathrm{Br}-\alpha$ WGT event (Figure 1). Six intra-cellular NHX genes were identified and the phylogentic analysis showed that there was one unique ortholog of each of the A. thaliana NHX1-6 genes (Figure 2B), indicating that there was an AtNHX5 ortholog present in the ancient Brassica ancestor species before the $\mathrm{Br}-\alpha$ WGT.

\section{MACRO- AND MICRO-SYNTENY OF ATNHX5 AND ATNHX6}

To be confident that the BrNHX6.1 and BrNHX6.2 genes identified are both orthologs of AtNHX6 and not AtNHX5, we analysed the macrosynentic relationship between these genes based on the 24 conserved syntenic blocks between A. thaliana and B. rapa (Schranz et al., 2006). Analysis of the conserved co-linearity in A. thaliana and B. rapa shows that BrNHX6.1, BrNHX6.2, and AtNHX6 are all located in the retained conserved genomic block $\mathrm{E}$ and are likely to be true orthologs, whereas AtNHX5 is retained within the conserved genomic Block $\mathrm{C}$ on the $A$. thaliana genome (Table 1), and appears to have no clear ortholog in B. rapa. We also analysed the gene models for AtNHX5, AtNHX6, BrNHX6.1, and BrNHX6.2 (Figure 3). This showed that AtNHX6, BrNHX6.1, and BrNHX6.2 all have a common 22 exon/21 intron gene structure, whereas AtNHX5 has only 21 exons (Figure 3) supporting the notion that the BrNHX6.1 and BrNHX6.2 genes are both orthologous to AtNHX6 and not AtNHX5.

Although it is clear that there was an AtNHX5 ortholog present prior to the Br- $\alpha$ WGT event, no AtNHX5 homolog was identified in the B. rapa genome. To determine the cause of this discrepancy we decided to carefully investigate the microsynteny around AtNHX5 (Figure 4A). The microsynteny in the A. thaliana genome, including fourteen genes downstream and twelve genes upstream of AtNHX5, was compared to the corresponding colinear sub genome regions in the B. rapa genome (Figure 4A). Surprisingly, the corresponding co-linear regions of the three B. rapa sub genomes contain only five (LF), three (MF1) and nine (MF2) genes respectively, and only four (LF), three (MF1) and five (MF2) of these genes were homologous to $A$. thaliana genes (Figure 4A). The colinearity between $A$. thaliana and the B. rapa sub genomes is relatively well conserved from the At1G54220 gene to At1G54290 (Figure 4A). However, the next 14 genes upstream of At1G54290 in the A. thaliana genome including AtNHX5 are completely absent from all three co-linear $B$. rapa sub genomes (Figure 4A). Co-linear genes are again found between the $A$. thaliana genome and the $B$. rapa LF sub genome from At1G54490 and the MF2 sub genomes from At1G5440, however, no homologous genes can be detected in the MF1 sub genome (Figure 4A). Interestingly, upstream of At1G54440 the co-linear region on the LF sub genome is found on chromosome BrA09, whereas the co-linear region of the LF sub genome downstream of At1G54290 was found on chromosome BrA06 (Figure 4A). Similarly, the region of co-linearity upstream of At1G54440 on the MF2 sub genome is found on chromosome BrA01 and the colinear region of the MF2 sub genome downstream of At1G54290 was found on chromosome BrA08 (Figure 4A). This indicates that the syntenic regions around AtNHX5 in the LF and MF2 sub genomes have probably undergone an inter-chromosomal translocation event. It also appears that there has been a large fragment deletion event in the MF1 sub genome in the syntenic region around AtNHX5. These chromosomal re-arrangments and deletions would account for the absence of any AtNHX5 orthologs in $B$. rapa and may be the result of the genome reduction processes post the $\mathrm{Br}-\alpha$ WGT event. This evidence, combined with the inability to identify any AtNHX5 orthologs when searching the $B$. rapa genome, strongly suggest that there are no AtNHX5 orthologs present in B. rapa.

The microsynteny in the $A$. thaliana genome, including eight genes downstream and eight genes upstream of AtNHX6, was compared to the corresponding co-linear sub genome regions in the $B$. rapa genome (Figure 4B). The corresponding three co-linear B. rapa sub genomes contain 13 (LF), 16 (MF1), and 10 (MF2) genes, respectively, with 11 (LF), nine (MF1), and 10 (MF2) homologous genes to the co-linear region in the A. thaliana genome (Figure 4B). The BrNHX6.1 gene is located on the LF sub genome and the BrNHX6.2 gene is located of 


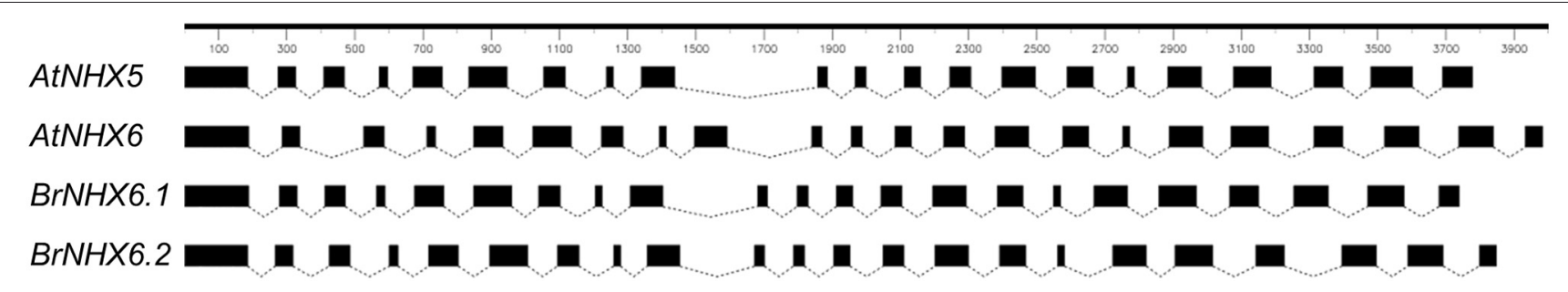

FIGURE 3 | Predicted gene models for AtNHX5, AtNHX6, BrNHX6.1 and BrNHX6.2. Predicted gene models for AtNHX5, AtNHX6, BrNHX6.1, and BrNHX6.2. Exons are shown as black boxes and introns are shown as dotted lines. Gene models were generated using FancyGene 1.4 (http://www.bio.ieo.eu/fancygene).
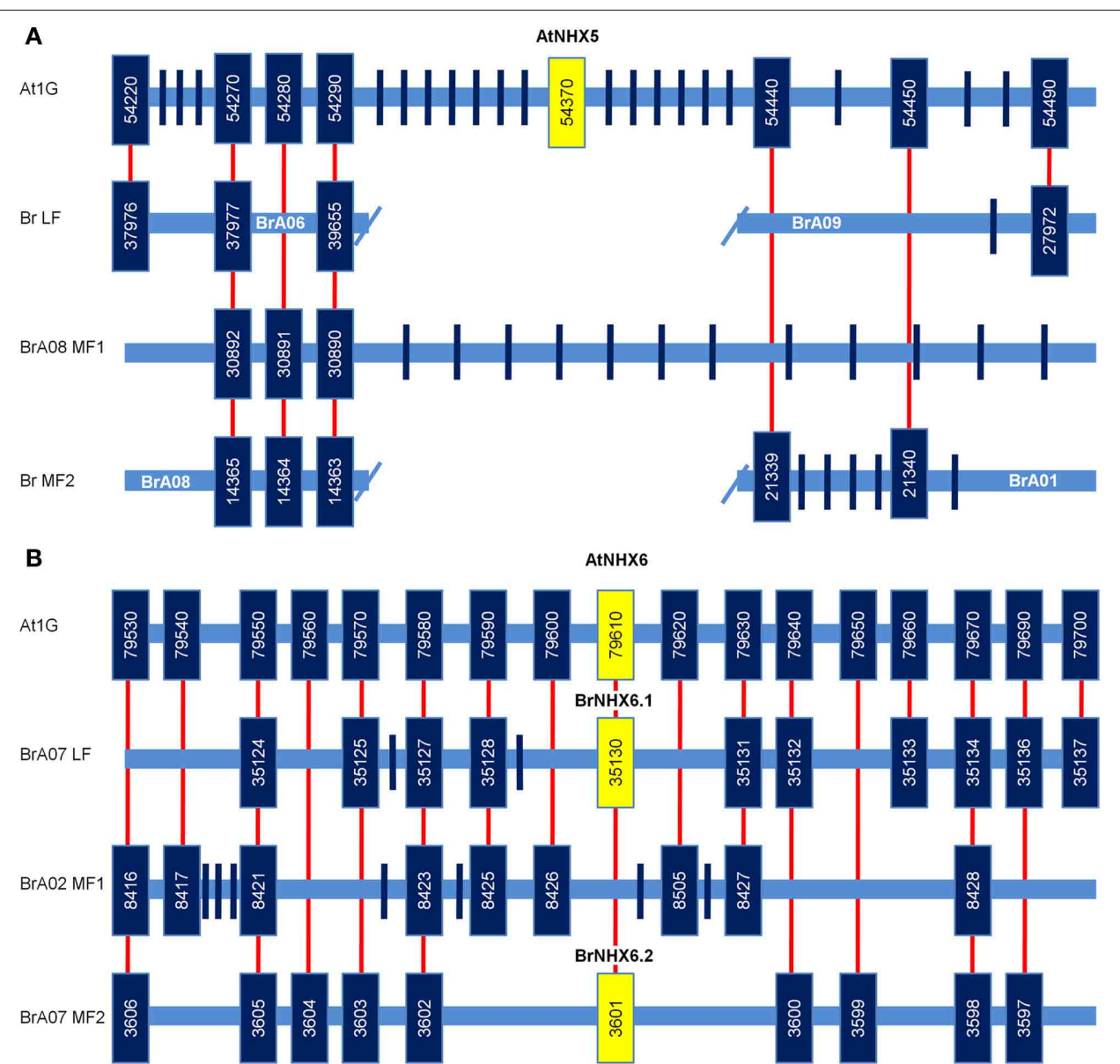

FIGURE 4 | Microsynteny of AtNHX5 and AtNHX6 and conserved co-linearity in B. rapa. Comparison of co-linearity between AtNHX5 (A) and AtNHX6 (B) (highlighted in yellow) in the Arabidopsis genome and the least fractionated (LF), medium fractionated (MF1) and most fractionated (MF2) sub-genomes in B. rapa. The boxes and bars denote annotated genes (boxes contain gene accession number) and the red lines connect syntenic homologous genes (Note corresponding genomic regions are not drawn to scale). the MF2 sub genome, but there is no AtNHX6 ortholog present on the MF1 sub genome (Figure 4B). Importantly, all of the 17 A. thaliana genes in the region surrounding AtNHX6 have at least one homolog in one of the corresponding $B$. rapa sub genomes and the order of the genes in A. thaliana is maintained within B. rapa (Figure 4B). The high degree of co-linearity between the region in A. thaliana genome containing AtNHX6 and the corresponding regions in the $B$. rapa genome strongly support the notion that there are only two AtNHX6 orthologs in B. rapa with the third ortholog likely to have been lost from the MF1 sub genome since the last whole genome triplication event.

\section{PROMOTER ANALYSIS OF BrNHX6.1, BrNHX6.2 AND AtNHX6}

Although the $B$. rapa genome does not appear to contain a homolog to AtNHX5, there is a high level of genetic conservation between the A. thaliana and B. rapa NHX6.1 and NHX6.2 


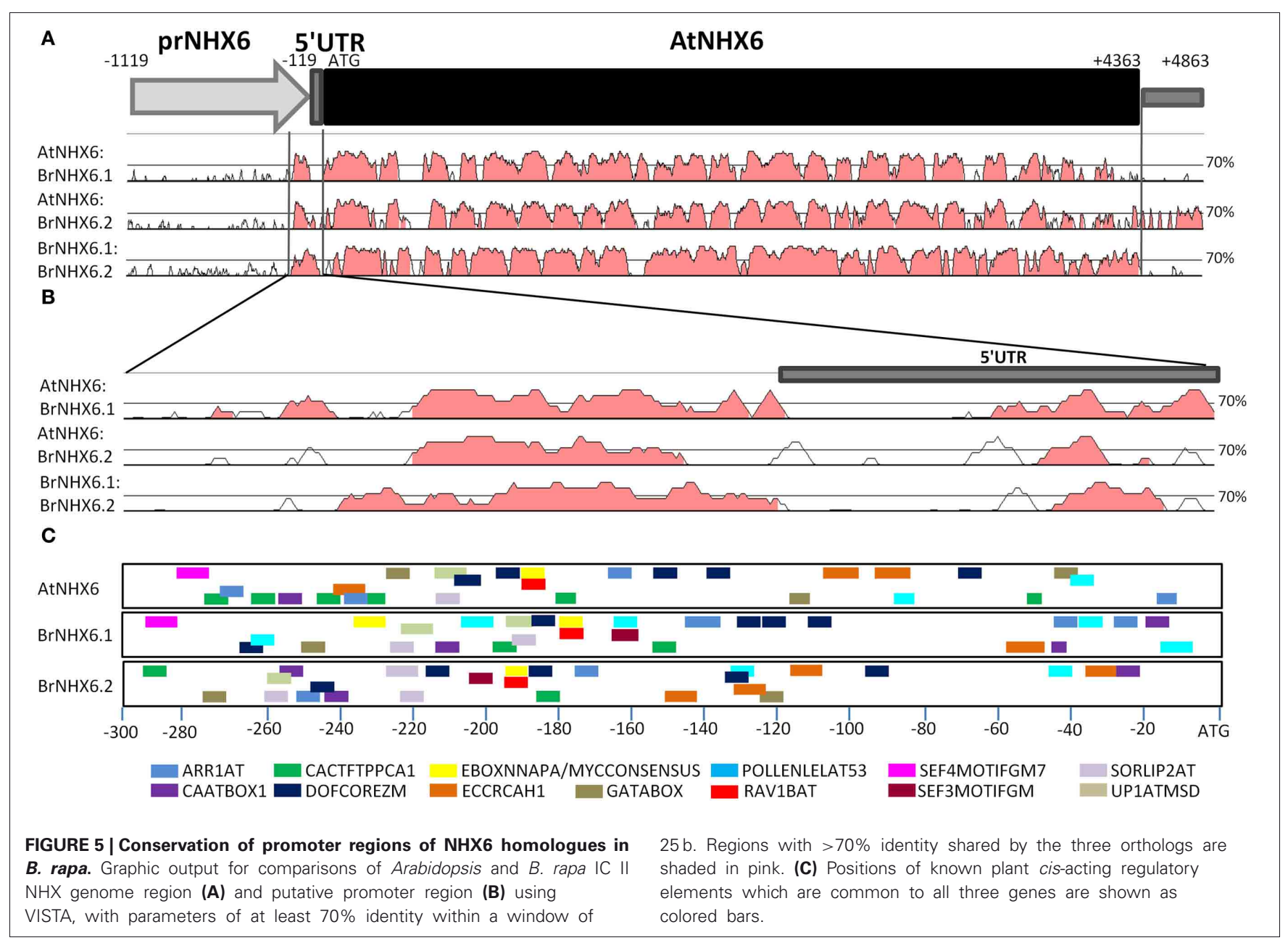

orthologs. To explore the potential for differential functionality between these two genes, we investigated the level of sequence similarity between their respective promoter regions and the conservation of specific cis-acting regulatory motifs.

To identify conserved regions within the promoters of $B$. rapa NHX6 orthologs, we compared nucleotide sequences of AtNHX6, BrNHX6.1, and BrNHX6.2 (Figure 5A). The gene sequences, including $1119 \mathrm{bp}$ upstream and $500 \mathrm{bp}$ downstream, were subjected to sliding-widow analysis of homology. It has previously been shown that requiring $70 \%$ identity within a 25 -bp window returns the greatest number of regulatory elements with acceptable specificity (Guo and Moose, 2003). Using these parameters, sliding window analysis revealed a highly conserved region of approximately $300 \mathrm{bp}$ immediately upstream of the start codon, including the $5^{\prime} \mathrm{UTR}$ (Figure 5B).

Evaluation of this highly conserved promoter region $(-300 \mathrm{bp})$ using the PLACE database SIGNALSCAN tool identified 10 cis-acting regulatory elements (CREs) which were common to all three genes: ARR1AT (5'-NGATT- $\left.3^{\prime}\right)$, CAATBOX1 (5'-CAAT- $\left.3^{\prime}\right)$, CACTFTPPCA1 (5'-YACT- $\left.3^{\prime}\right)$, DOFCOREZM ( $5^{\prime}$-AAAG- $\left.3^{\prime}\right)$, EBOXNNAPA ( $5^{\prime}$-CANNAG- $\left.3^{\prime}\right)$, EECCRCAH1 (5'-GANTTNC- $\left.3^{\prime}\right)$, GATABOX (5'-GATA$\left.3^{\prime}\right)$, MYCCONSENSUS (5'-CANNAG-3'), POLLENLELAT53
(5'-AGAAA-3'), RAV1BAT (5'-CACCTG-3'), SORLIP2AT (5'-GC CAC- $\left.3^{\prime}\right)$, and UP1ATMSD ( $5^{\prime}$-GGCCCAWWW-3'). Additionally, the functionally similar elements SEF4MOTIFGM7S (5'-RTTTTTR-3') and SEF3MOTIFGM (5'-AACCCA$\left.3^{\prime}\right)$ were conserved between AtNHX6:BrNHX6.1 and BrNHX6.1:BrNHX6.2, respectively. The position of these motifs in the promoter sequence relative to the start codon is depicted in Figure 5C. Interestingly, the position of the DOFCO REZM (5'-AAAG- $\left.3^{\prime}\right)$ and MYCCONSENSUS/EBOXNNAPA $\left(5^{\prime}\right.$-CANNAG-3')/RAV1BAT $\left(5^{\prime}\right.$-CACCAG- $\left.3^{\prime}\right)$ elements are conserved across the three promoter regions.

\section{IDENTIFICATION B. napus NHX6 HOMOLOGS}

B. napus is an agriculturally important member of the Brassicaceae family. Due to the significance of salinity in B. napus cultivation, we sought to identify the complement of expressed B. napus NHX6 homologs and assess their role in salt tolerance. To identify NHX6 homologs in B. napus without the advantage of a complete $B$. napus genome sequence, we designed primers in absolutely conserved regions present in all available nucleotide sequences from $B$. napus, B. rapa, and B. oleracea. These primers were first tested on $B$. rapa genomic DNA to ensure they could identify both BrNHX6.1 and BrNHX6.2. Using these 
"universal” Brassica NHX6 primers, four unique sequences were identified in B. rapa. A phylogenetic analysis of these sequences revealed two distinct groups including either the BrNHX6.1 or the BrNHX6.2 sequences from the B. rapa genome (Figure 6A). The presence of two unique sequences grouping with each $\mathrm{BrNHX6}$ sequence is most likely the result of heterozygous $B r N H X 6$ alleles present in the B. rapa genomic DNA tested. Importantly, however, the primers used were able to amplify both the BrNHX6 homologs.

The complement of expressed AtNHX6 homologs in B. napus was then investigated using the same approach, but using mRNA isolated from B. napus seedlings. Sequencing the cloned amplicons from $B$. napus cDNA identified only two unique sequences. A phylogenetic analysis was performed including BrNHX6.1 and BrNHX6.2 sequences from the B. rapa genome, the B. napus (JX082291) and B. oleracea (JX082292) ORF sequences and a $B$. oleracea (DK554651) EST sequence. The phylogenetic analysis showed two major groupings, each containing either the BrNHX6.1 or the BrNHX6.2 sequence (Figure 6B). Interestingly, only one sequence grouped with BrNHX6.2 while four unique sequences grouped with BrNHX6.1 (Figure 6B). The sequences that grouped with $B r N H X 6.1$ were divided into two sub-groups-one group has a $B$. napus sequence more closely related to the BrNHX6.1 sequence, while the other group includes only $B$. oleracea and $B$. napus sequences, possibly indicating both an $\mathrm{A}$ and $\mathrm{C}$ genome version of BrNHX6.1 (Figure 6B). From this analysis, the B. napus sequences that are clearly closely related to BrNHX6.1 and BrNHX6.2 will be hereafter designated as $B n N H X 6.1$ and $B n N H X 6.2$, respectively.

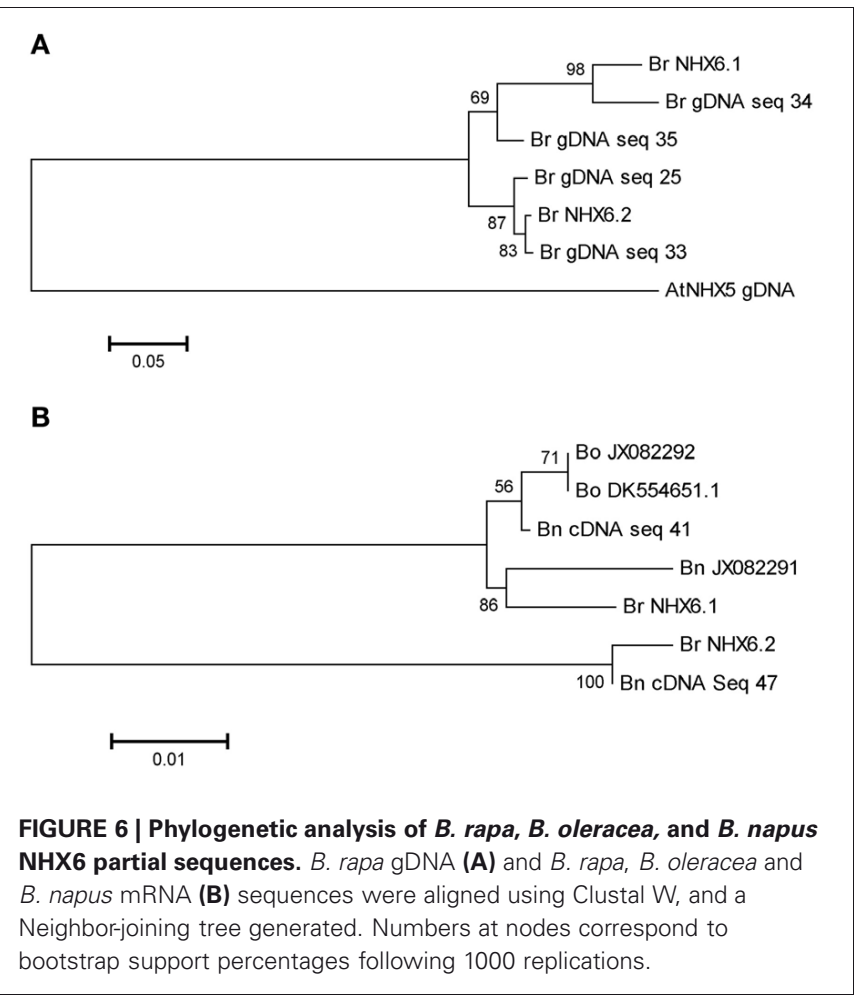

\section{EXPRESSION ANALYSIS OF BnNHX6.1 AND BnNHX6.2}

Having identified two NHX6 genes in B. napus, we examined the relative expression of $B n N H X 6.1$ and $B n N H X 6.2$ in the presence and absence of salt stress. Firstly, to optimise the concentration of $\mathrm{NaCl}$ required to induce salt stress, we compared the germination percentage and fresh weight of $B$. napus $\mathrm{cv}$. Westar seedlings grown in the presence and the absence of $\mathrm{NaCl}$. The $\mathrm{NaCl}$ treatment had a marked effect on the germination and growth of B. napus seedlings (Figure 7A). Germination in the presence of $\mathrm{NaCl}$ was severely inhibited with only $41 \%$ of seeds germinating compared with $92 \%$ of seeds sown on plates lacking additional $\mathrm{NaCl}$ (Figure 7B). The mean fresh weight per seedling grown in the presence of $\mathrm{NaCl}$ was also greatly reduced compared to those grown in the absence of $\mathrm{NaCl}$ (Figure 7C). These results demonstrate that the $\mathrm{NaCl}$ treatment was sufficient to induce a severe stress impacting on the germination and growth of $B$. napus seedlings. We then examined the differential expression of both the BnNHX6.1 and the BnNHX6.2 genes in the presence and absence of the $\mathrm{NaCl}$ stress. The BnNHX6.2 gene showed no significant difference in relative transcript abundance in response to the $\mathrm{NaCl}$ stress, whereas there was a significant increase in relative transcript abundance of the BnNHX6.1 gene in response to the $\mathrm{NaCl}$ stress (Figure 7D). Interestingly, the relative expression of BnNHX6.1 was extremely low compared to BnNHX6.2 both in the presence and absence of $\mathrm{NaCl}$ (Figure 7D) suggesting that there may be some differential regulation of these two genes.

\section{DISCUSSION \\ ORTHOLOG IDENTIFICATION IN B. napus}

In this paper we have attempted to identify the B. napus orthologs of two A. thaliana proteins, AtNHX5 and AtNHX6. Although $A$. thaliana and $B$. napus are relatively closely related, precise ortholog identification is complicated by the recent WGT event in the Brassica species and the amphidiploid nature of the B. napus (AACC $2 n=38$ ) genome. Consequently, there are potentially six B. napus homologs of AtNHX5 and AtNHX6 comprising three triplicated paralogs from both the A and $\mathrm{C}$ parental genomes.

Identification of $A$. thaliana homologs in the Brassica species has traditionally relied upon comparison to incomplete genomic sequences, generally in the form of bacterial artificial chromosome (BAC) clones. For example identification of FLOWERING LOCUS C (FLC) homologs in B. rapa entailed probing B. rapa BAC libraries to identify BAC clones containing co-linear regions (Yang et al., 1999). While successful in this case, this methodology was reliant on having all the appropriate co-linear region of the $B$. rapa genome present in the BAC library. The recent release of the $B$. rapa genome provides a complete Brassica genomic resource allowing $A$. thaliana gene orthologs to be identified with a high level of confidence.

Our search of the B. rapa genome identified only two AtNHX6 orthologs. Analysis of the B. rapa genome has shown that there has been significant loss of gene paralogs in the three sub genomes since the WGT event (Wang et al., 2011), which is likely to be the reason that there is no AtNHX6 ortholog on the MF1 sub genome. Interestingly, we could not find any evidence of an AtNHX5 homolog in the $B$. rapa genome. While this came as a surprise, recent evidence shows that $A t N H X 5$ and AtNHX6 act redundantly 


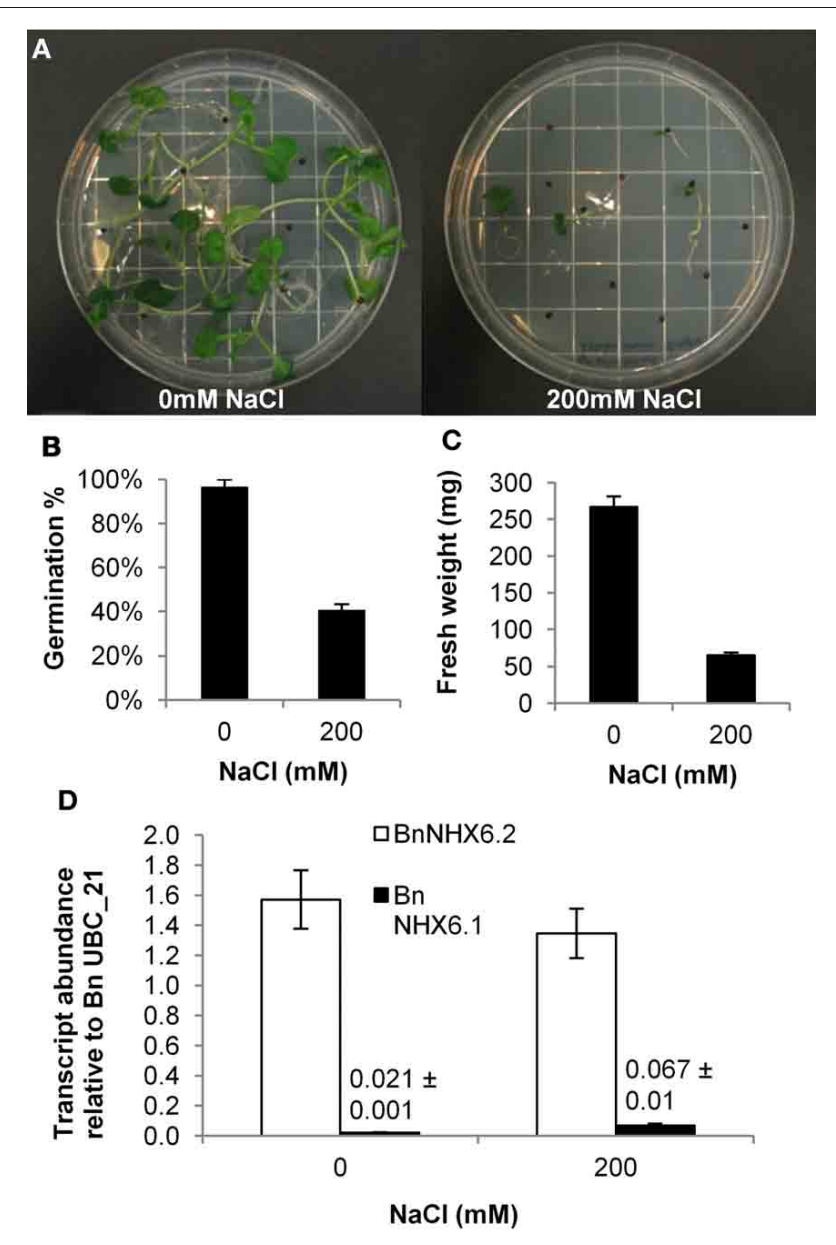

FIGURE 7 | Differential expression of BnNHX6.1 and BnNHX6.2 in B. napus seedlings in response to $\mathrm{NaCl}$. B. napus (cv Westar) seeds were sown on $1 / 2$ strength $\mathrm{MS}$ media with or without $200 \mathrm{mM} \mathrm{NaCl}$ (A) and the germination percentage (B) and fresh weight per seedling (C) were compared between the two treatments. RNA was extracted from seedlings and the transcript abundance of $\mathrm{BnNHX6.1}$ and $\mathrm{BnNHX6.2}$ was assayed relative to the BnUBC_21 reference gene (D). Data are the means of three biological replicates ( \pm s.e.).

in A. thaliana (Bassil et al., 2011), and therefore the the Brassica species may not require a functional AtNHX5 ortholog. Our analysis of the microsynteny around AtNHX5 and its co-linearity with the three corresponding sub genomes in $B$. rapa indicated that a large block of $A$. thaliana genes including AtNHX5 were missing from all three co-linear B. rapa sub genomes as a result of interchromosomal translocations in the LF and MF2 sub genomes and a fragment deletion in the MF1 sub-genome. The identification of an AtNHX5 ortholog in the closely related T. parvula species indicates that there was an AtNHX5 ortholog present in the ancient Brassica ancestral species prior to the $\mathrm{Br}-\alpha$ WGT event. The loss of AtNHX5 in the Brassica species could only have occurred either due to a localized deletion event occurring in the ancestral Brassica genome after the T. parvula-Brassica divergence, but before the Brassica WGT event or due to the loss of all three AtNHX5 genes following the WGT event. It seems more likely that the AtNHX5 orthologs were lost after the $\mathrm{Br}-\alpha$ WGT event due to the differential disruption in the syntentic region around AtNHX5 in the three B. rapa sub-genomes. If AtNHX5 had been lost prior to the $\mathrm{Br}-\alpha$ WGT event it would be reasonable to expect that the syntenic region in all three $B$. rapa sub-genomes would display a common chromosomal disruption that was then triplicated as part of the $\mathrm{Br}-\alpha$ WGT event.

Sequencing of orthologous BAC clones of the B. rapa $\mathrm{A}$ genome, the $B$. oleracea $\mathrm{C}$ genome and the $\mathrm{A}$ and $\mathrm{C}$ genome components of $B$. napus have shown essentially complete co-linearity between the protein coding genes in the $\mathrm{A}$ and $\mathrm{C}$ genomes and sequence identity of $94-97 \%$ (Cheung et al., 2009). It could, therefore, be anticipated that the B. oleracea $\mathrm{C}$ genome is also likely to only have two AtNHX6 orthologs. Our sequence analysis in $B$. oleracea and $B$. napus could only identify two distinct AtNHX6 homologs that were also homologous to the BrNHX6.1 and BrNHX6.2 genes. It is important to note however, that all of the $B$. oleracea and $B$. napus sequences analysed in this phylogeny were derived from mRNA; it is therefore possible that additional B. oleracea NHX6 homologs are present in the genome but have not been detected in the limited mRNA sequencing described here. The complete complement of B. oleracea NHX6 homologs could only be resolved with a completed $B$. oleracea genome. The phylogenetic analysis of homologous Brassica NHX6.1 sequences shows two distinct groupings, one including B. rapa NHX6.1 and a $B$. napus sequence, and the other containing only $B$. oleracea and $B$. napus sequences. This provides evidence of possible A and $\mathrm{C}$ genome versions of Brassica NHX6.1. Unfortunately, we do not have a corresponding B. oleracea NHX6.2 sequence available, making it difficult to clearly identify the $\mathrm{A}$ and $\mathrm{C}$ genome versions of NHX6.2. It is therefore most likely that there are two AtNHX6 homologs in both $B$. rapa and B. oleracea and, as a consequence, four AtNHX6 homologs in B. napus.

\section{EXPRESSION PATTERNS OF BnNHX6.1 AND BnNHX6.2}

The analysis of the $B$. rapa genome showed that of the eight Arabidopsis NHX genes only AtNHX1 and AtNHX6 have retained multiple paralogs since the most recent triplication event. Gene duplications arising from polyploidy events are thought to be a major source of novel variation and gene function (Moore and Purugganan, 2003) and duplicated genes may be lost, develop new functions or share the functions of the original gene through differential expression or regulation (Blanc and Wolfe, 2004). It may be the case that the two orthologs of AtNHX6 may have different functions or expression patterns. As the coding regions of all the identified Brassica NHX6 genes are highly homologous with AtNHX6 and each other, it is likely that their functions $\left(\mathrm{Na}^{+} / \mathrm{K}^{+}-\mathrm{H}^{+}\right.$antiporting) remain identical. The analysis of the BrNHX6.1 and BrNHX6.2 promoter regions highlighted a significant region of similarity $300 \mathrm{bp}$ upstream of the ATG. The conserved MYCCONSENSUS element is often associated with genes responsive to abiotic stress, such as the stress hormone abscisic acid (ABA), dehydrationresponsive $\mathrm{RD} 22$, and cold responsive genes CBF, DREB1, and ICE1 (Chinnusamy et al., 2004). This is highly interesting given the potential role for $N H X$ proteins in improving salt tolerance. Additionally, the equivalent EBOXNNAPA motif was found 
to be an essential promoter element for high expression of the napA storage protein in B. napus seeds (Stalberg et al., 1996). The conservation of these regulatory elements suggests conservation of functionality between AtNHX6 and its B. rapa homologs.

The expression of the B. napus NHX6.2 gene is significantly higher than the B. napus NHX6.1 gene in two week old seedlings, indicating that in this tissue type at least there does appear to be some differential expression. While there is no significant difference in expression of $B n N H X 6.2$ in response to $\mathrm{NaCl}$, the expression of $B n N H X 6.1$ increased approximately three-fold in response to $\mathrm{NaCl}$ again highlighting differences in expression. It should be noted that the relative expression of BnNHX6.1 was 20 times lower than the expression of $B n N H X 6.2$ in the $\mathrm{NaCl}$ treatment, and is suggestive of a more prominent role for BnNHX6.2. This is in contrast to the situation in A. thaliana, where the largest difference in expression observed is a 3.5 increase of AtNHX5 transcript in leaf tissue (Bassil et al., 2011). The two Brassica NHX6 isoforms do not appear to have different functions, but the expression of BnNHX6.1 is significantly lower than that of $B n N H X 6.2$. This may indicate that the BnNHX6.1 gene is simply

\section{REFERENCES}

Apse, M. P., Aharon, G. S., Snedden, W. A., and Blumwald, E. (1999). Salt tolerance conferred by overexpression of a vacuolar $\mathrm{Na}+/ \mathrm{H}+$ antiport in Arabidopsis. Science 285, 1256-1258.

Bassil, E., Ohto, M. A., Esumi, T., Tajima, H., Zhu, Z., Cagnac, O., Belmonte, M., Peleg, Z., Yamaguchi, T., and Blumwald, E. (2011). The Arabidopsis intracellular $\mathrm{Na}+/ \mathrm{H}+$ antiporters NHX5 and NHX6 are endosome associated and necessary for plant growth and development. Plant Cell 23, 224-239.

Bell, C. D., Soltis, D. E., and Soltis, P. S. (2010). The age and diversification of the angiosperms re-revisited. Am. J. Bot. 97, 1296-1303.

Blanc, G., and Wolfe, K. H. (2004). Functional divergence of duplicated genes formed by polyploidy during Arabidopsis evolution. Plant Cell 16, 1679-1691.

Bowers, K., Levi, B. P., Patel, F. I., and Stevens, T. H. (2000). The sodium/proton exchanger Nhxlp is required for endosomal protein trafficking in the yeast Saccharomyces cerevisiae. Mol. Biol. Cell 11, 4277-4294.

Brett, C. L., Donowitz, M., and Rao, R. (2005a). Evolutionary origins of eukaryotic sodium/proton exchangers. Am. J. Physiol. Cell Physiol 288, C223-C239.

Brett, C. L., Tukaye, D. N., Mukherjee, S., and Rao, R. J. (2005b). The yeast endosomal $\mathrm{Na}+(\mathrm{K}+) / \mathrm{H}+$ exchanger Nhx1 regulates cellular $\mathrm{pH}$ to control vesicle trafficking. Mol. Biol. Cell 16, 1396-1405.
Chanroj, S., Wang, G., Venema, K., Zhang, M. W., Delwiche, C. F., and Sze, H. (2012). Conserved and diversified gene families of monovalent cation $/ \mathrm{H}^{(+)}$antiporters from algae to flowering plants. Front. Plant Science 3:25. doi:

Chen, X., Truksa, M., Shah, S., and Weselake, R. J. (2010). A survey of quantitative real-time polymerase chain reaction internal reference genes for expression studies in Brassica napus. Anal. Biochem. 405, 138-140.

Cheung, F., Trick, M., Drou, N., Lim, Y. P., Park, J. Y., Kwon, S. J., Kim, J. A., Scott, R., Pires, J. C., Paterson, A. H., Town, C., and Bancroft, I. (2009). Comparative analysis between homoeologous genome segments of Brassica napus and its progenitor species reveals extensive sequence-level divergence. Plant Cell 21, 1912-1928.

Chinnusamy, V., Schumaker, K., and Zhu, J. K. (2004). Molecular genetic perspectives on cross-talk and specificity in abiotic stress signalling in plants. J. Exp. Bot. 55, 225-236.

Couvreur, T. L., Franzke, A., AlShehbaz, I. A., Bakker, F. T., Koch, M. A., and Mummenhoff, K. (2010). Molecular phylogenetics, temporal diversification, and principles of evolution in the mustard family (Brassicaceae). Mol. Biol. Evol. 27, 55-71.

Dassanayake, M., Oh, D. H., Haas, J. S., Hernandez, A., Hong, H., Ali, S., Yun, D. J., Bressan, R. A., Zhu, J. K., Bohnert, H. J., and Cheeseman, 10.3389/fpls.2012.00025

sitting in the genome waiting to be lost, like most of the other triplicated paralogs of the BrNHX gene family. It would be of great interest to examine the expression patterns of BnNHX6.1 and $B n N H X 6.2$ in a variety of tissues types in the presence and absence of $\mathrm{NaCl}$ to better elucidate differential expression patterns.

In this study we have demonstrated the use of the $B$. rapa genome in the successful identification of two B. rapa NHX6 orthologs, and used this information to identify two unique B. napus NHX6 orthologs. Our analysis also strongly suggests that there are four B. napus orthologs, comprising an $\mathrm{A}$ and $\mathrm{C}$ genome version of BrNHX6.1 and BrNHX6.2. This study also indicates that there may be some differential expression of the BnNHX6.1 and BnNHX6.2.

\section{ACKNOWLEDGMENTS}

This work was supported by the Grains Research Development Corporation through a Undergraduate Honours Scholarship (UHS119) and a Graduate Research Scholarship (GRS161) to Brett A. Ford and GRS179 to Joanne R. Ernest.

J. M. (2011). The genome of the extremophile crucifer Thellungiella parvula. Nat. Genet. 43, 913-918.

Food, and Agriculture Organization of the United Nations. (2009). "Increasing crop production sustainably. The perspective biological processes," ed Department of Economic and Social Development (Rome: FAO).

Franzke, A., Lysak, M. A., Al-Shehbaz, I. A., Koch, M. A., and Mummenhoff, K. (2011). Cabbage family affairs: the evolutionary history of Brassicaceae. Trends Plant Sci. $16,108-116$.

Frazer, K. A., Pachter, L., Poliakov, A., Rubin, E. M., and Dubchak, I. (2004). VISTA: computational tools for comparative genomics. Nucleic Acids Res. 32, W273-W279.

Fukuda, A., Nakamura, A., Hara, N., Toki, S., and Tanaka, Y. (2011). Molecular and functional analyses of rice NHX-type $\mathrm{Na}^{+} / \mathrm{H}^{+}$ antiporter genes. Planta 233, 175-188.

Goodstein, D. M., Shu, S., Howson, R., Neupane, R., Hayes, R. D., Fazo, J., Mitros, T., Dirks, W., Hellsten, U., Putnam, N., and Rokhsar, D. S. (2012). Phytozome: a comparative platform for green plant genomics. Nucleic Acids Res. 40, D1178-D1186.

Guo, H., and Moose, S. P. (2003). Conserved noncoding sequences among cultivated cereal genomes identify candidate regulatory sequence elements and patterns of promoter evolution. Plant Cell 15, 1143-1158.
Henry, Y., Bedhomme, M., and Blanc, G. (2006). History, protohistory and prehistory of the Arabidopsis thaliana chromosome complement. Trends Plant Sci. 11, 267-273.

Herrmann, B. G., and Frischauf, A. M. (1987). Isolation of genomic DNA. Methods Enzymol. 152, 180-183.

Higo, K., Ugawa, Y., Iwamoto, M., and Korenaga, T. (1999). Plant cis-acting regulatory DNA elements (PLACE) database: 1999. Nucleic Acids Res. 27, 297-300.

$\mathrm{Hu}$, T. T., Pattyn, P., Bakker, E. G., Cao, J., Cheng, J. F., Clark, R. M., Fahlgren, N., Fawcett, J. A., Grimwood, J., Gundlach, H., Haberer, G., Hollister, J. D., Ossowski, S., Ottilar, R. P., Salamov, A. A., Schneeberger, K., Spannagl, M., Wang, X., Yang, L., Nasrallah, M. E., Bergelson, J., Carrington, J. C., Gaut, B. S., Schmutz, J., Mayer, K. F., Van De Peer, Y., Grigoriev, I. V., Nordborg, M., Weigel, D., and Guo, Y. L. (2011). The Arabidopsis lyrata genome sequence and the basis of rapid genome size change. Nat. Genet. 43, 476-481.

Koch, M. A., Haubold, B., and MitchellOlds, T. (2000). Comparative evolutionary analysis of chalcone synthase and alcohol dehydrogenase loci in Arabidopsis, Arabis, and related genera (Brassicaceae). Mol. Biol. Evol. 17, 1483-1498.

Li, M., Li, Y., Li, H., and Wu, G. (2011). Overexpression of AtNHX5 improves tolerance to both salt and drought stress in Broussonetia 
papyrifera (L.) Vent. Tree Physiol. 31, 349-357.

Lysak, M. A., Koch, M. A., Pecinka, A., and Schubert, I. (2005). Chromosome triplication found across the tribe Brassiceae. Genome Res. 15, 516-525.

Moore, R. C., and Purugganan, M. D. (2003). The early stages of duplicate gene evolution. Proc. Natl. Acad. Sci. U.S.A. 100, 15682-15687.

Mun, J. H., Kwon, S. J., Yang, T. J., Seol, Y. J., Jin, M., Kim, J. A., Lim, M. H., Kim, J. S., Baek, S., Choi, B. S., Yu, H. J., Kim, D. S., Kim, N., Lim, K. B., Lee, S. I., Hahn, J. H., Lim, Y. P., Bancroft, I., and Park, B. S. (2009). Genome-wide comparative analysis of the Brassica rapa gene space reveals genome shrinkage and differential loss of duplicated genes after whole genome triplication. Genome Biol. 10, R111.

Munns, R., and Tester, M. (2008). Mechanisms of salinity tolerance. Annu. Rev. Plant Biol. 59, 651-681.

Murashige, T., and Skoog, F. (1962). A revised medium for rapid growth and bio-assays with tobacco tissue cultures. Physiol. Plant 15, 473-497.

Parkin, I. A., Gulden, S. M., Sharpe, A. G., Lukens, L., Trick, M., Osborn, T. C., and Lydiate, D. J. (2005). Segmental structure of the Brassica napus genome based on comparative analysis with Arabidopsis thaliana. Genetics 171, 765-781.

Pfaffl, M. (2004). "Quantification strategies in real-time PCR," in $A-Z$ of quantitative PCR, ed S. Bustin (La Jolla, CA: International University Line), 87-112.

Rambaldi, D., and Ciccarelli, F. D. (2009). FancyGene: dynamic visualization of gene structures and protein domain architectures on genomic loci. Bioinformatics 25, 2281-2282.
Rodriguez-Rosales, M. P., Jiang, X., Galvez, F. J., Aranda, M. N., Cubero, B., and Venema, K. (2008). Overexpression of the tomato $\mathrm{K}^{+} / \mathrm{H}^{+}$antiporter LeNHX2 confers salt tolerance by improving potassium compartmentalization. New Phytol. 179, 366-377.

Schranz, M. E., Lysak, M. A., and Mitchell-Olds, T. (2006). The ABC's of comparative genomics in the Brassicaceae: building blocks of crucifer genomes. Trends Plant Sci. 11, 535-542.

Shi, H., Lee, B. H., Wu, S. J., and Zhu, J. K. (2003). Overexpression of a plasma membrane $\mathrm{Na}^{+} / \mathrm{H}^{+}$ antiporter gene improves salt tolerance in Arabidopsis thaliana. Nat. Biotechnol. 21, 81-85.

Shi, H., Quintero, F. J., Pardo, J. M., and Zhu, J. K. (2002). The putative plasma membrane $\mathrm{Na}(+) / \mathrm{H}(+)$ antiporter SOS1 controls longdistance $\mathrm{Na}(+)$ transport in plants. Plant Cell 14, 465-477.

Shi, H., and Zhu, J. K. (2002). SOS4, a pyridoxal kinase gene, is required for root hair development in Arabidopsis. Plant Physiol. 129, 585-593.

Soltis, D. E., Albert, V. A., LeebensMack, J., Bell, C. D., Paterson, A. H., Zheng, C., Sankoff, D., Depamphilis, C. W., Wall, P. K., and Soltis, P. S. (2009). Polyploidy and angiosperm diversification. Am. J. Bot. 96, 336-348.

Stalberg, K., Ellerstom, M., Ezcurra, I., Ablov, S., and Rask, L. (1996). Disruption of an overlapping Ebox/ABRE motif abolished high transcription of the napA storageprotein promoter in transgenic Brassica napus seeds. Planta 199, 515-519.

Tamura, K., Peterson, D., Peterson, N., Stecher, G., Nei, M., and Kumar,
S. (2011). MEGA5, molecular evolutionary genetics analysis using maximum likelihood, evolutionary distance, and maximum parsimony methods. Mol. Biol. Evol. 28, 2731-2739.

Tester, M., and Davenport, R. (2003). $\mathrm{Na}^{+}$tolerance and $\mathrm{Na}^{+}$transport in higher plants. Ann. Bot. (Lond.) 91, 503-527.

United States Department of Agriculture. (2009). "Oilseeds: world market and trade," ed Foreign Agricultural Service Circular Series 11-09 - November, (Washington, DC: USDA).

Wang, X., Wang, H., Wang, J., Sun, R., Wu, J., Liu, S., Bai, Y., Mun, J. H., Bancroft, I., Cheng, F., Huang, S., Li, X., Hua, W., Freeling, M., Pires, J. C., Paterson, A. H., Chalhoub, B., Wang, B., Hayward, A., Sharpe, A. G., Park, B. S., Weisshaar, B., Liu, B., Li, B., Tong, C., Song, C., Duran, C., Peng, C., Geng, C., Koh, C., Lin, C., Edwards, D., Mu, D., Shen, D., Soumpourou, E., Li, F., Fraser, F., Conant, G., Lassalle, G., King, G. J., Bonnema, G., Tang, H., Belcram, H., Zhou, H., Hirakawa, H., Abe, H., Guo, H., Jin, H., Parkin, I. A., Batley, J., Kim, J. S., Just, J. Li, J., Xu, J., Deng, J., Kim, J. A., Yu, J., Meng, J., Min, J., Poulain, J., Hatakeyama, K., Wu, K., Wang, L., Fang, L., Trick, M., Links, M. G., Zhao, M., Jin, M., Ramchiary, N., Drou, N., Berkman, P. J., Cai, Q., Huang, Q., Li, R., Tabata, S., Cheng, S., Zhang, S., Sato, S., Sun, S., Kwon, S. J., Choi, S. R., Lee, T. H., Fan, W., Zhao, X., Tan, X., Xu, X., Wang, Y., Qiu, Y., Yin, Y., Li, Y., Du, Y., Liao, Y., Lim, Y., Narusaka, Y., Wang, Z., Li, Z., Xiong, Z., and Zhang, Z. (2011). The genome of the mesopolyploid crop species Brassica rapa. Nat. Genet. 43, 1035-1039.
Yang, Y. W., Lai, K. N., Tai, P. Y., and Li, W. H. (1999). Rates of nucleotide substitution in angiosperm mitochondrial DNA sequences and dates of divergence between Brassica and other angiosperm lineages. J. Mol. Evol. 48, 597-604.

Yokoi, S., Quintero, F. J., Cubero, B., Ruiz, M. T., Bressan, R. A., Hasegawa, P. M., and Pardo, J. M. (2002). Differential expression and function of Arabidopsis thaliana NHX Na ${ }^{+} / \mathrm{H}^{+}$antiporters in the salt stress response. Plant J. 30, 529-539.

Conflict of Interest Statement: The authors declare that the research was conducted in the absence of any commercial or financial relationships that could be construed as a potential conflict of interest.

Received: 31 May 2012; accepted: 16 August 2012; published online: 11 September 2012.

Citation: Ford BA, Ernest JR and Gendall AR (2012) Identification and characterization of orthologs of AtNHX5 and AtNHX6 in Brassica napus. Front. Plant Sci. 3:208. doi: 10.3389/fpls. 2012.00208

This article was submitted to Frontiers in Plant Genetics and Genomics, a specialty of Frontiers in Plant Science.

Copyright (c) 2012 Ford, Ernest and Gendall. This is an open-access article distributed under the terms of the Creative Commons Attribution License, which permits use, distribution and reproduction in other forums, provided the original authors and source are credited and subject to any copyright notices concerning any third-party graphics etc. 